\title{
Lidil
}

Revue de linguistique et de didactique des langues

\section{L'incidence de la culture et de la langue dans les récits parlés et les gestes d'enfants français, italiens et américains âgés de 6 et 10 ans}

Olga Capirci, Jean-Marc Colletta, Carla Cristilli, Özlem Ece Demir, Michèle Guidetti et Susan Levine

\section{(2) OpenEdition}

Édition électronique

URL : http://journals.openedition.org/lidil/3078

DOI : 10.4000/lidil.3078

ISSN : 1960-6052

Éditeur

UGA Éditions/Université Grenoble Alpes

\section{Édition imprimée}

Date de publication : 30 novembre 2010

Pagination : 139-158

ISBN : 978-2-84310-184-7

ISSN : $1146-6480$

Référence électronique

Olga Capirci, Jean-Marc Colletta, Carla Cristilli, Özlem Ece Demir, Michèle Guidetti et Susan Levine, " L'incidence de la culture et de la langue dans les récits parlés et les gestes d'enfants français, italiens et américains âgés de 6 et 10 ans », Lidil [En ligne], 42 | 2010, mis en ligne le 31 mai 2012, consulté le 01 mai 2019. URL : http://journals.openedition.org/lidil/3078 ; DOI : 10.4000/lidil.3078 


\title{
L'incidence de la culture et de la langue dans les récits parlés et les gestes d'enfants français, italiens et américains âgés de 6 et 10 ans
}

\author{
Olga Capirci*, Jean-Marc Colletta**, Carla Cristilli***, \\ Özlem Ece Demir****, Michèle Guidetti***** \\ et Susan Levine $* * * *$
}

\begin{abstract}
RÉSUMÉ
La culture, la langue et l'âge ont une incidence sur les conduites langagières en général. Dans le cas présent, nous avons voulu vérifier de quelle façon les conduites narratives multimodales (paroles et gestes) sont sensibles à ces trois facteurs, afin de mieux apprécier le rôle respectif des acquisitions linguistiques et du développement cognitif dans l'évolution des capacités discursives chez l'enfant. L'étude porte sur plus de 150 récits parlés produits par des enfants français, italiens et américains, filmés puis transcrits à l'aide d'un logiciel d'annotation multimodale. Les premiers résultats font apparaitre des différences tant au plan linguistique qu'au plan gestuel. Ce sont les enfants français qui réalisent les récits les plus longs et détaillés, mais ce sont les enfants italiens qui utilisent le mieux les ressources gestuelles pour les mettre au service de la construction référentielle. Quant à l'effet de l'âge, il est visible sur l'ensemble des variables linguistiques en même temps que sur les aspects gestuels de la cohésion discursive et de la représentation des évènements. Il semble qu'en dépit des différences inhérentes aux langues et des variations culturelles en matière de gestualité, l'évolution des capacités discursives et des capacités cognitives sous-jacentes soit semblable dans les trois cas.
\end{abstract}

\footnotetext{
* Gesture \& Language Lab, ICST, CNR, Rome.

** LIDILEM, Université de Grenoble.

*** Università degli Studi di Napoli «L'Orientale», Naples.

**** Department of Psychology, University of Chicago.

****** Laboratoire Octogone, Université de Toulouse.
} 


\section{ABSTRACT}

It has been demonstrated that culture, language and age have an effect on language behavior. In this paper, we study the way speech and gesture narrative behavior respond to these three factors in order to better understand the respective role of linguistic acquisition and cognitive development in the building of monologic discourse abilities. We asked over 150 six years old and ten years old French, Italian and American children to narrate from a short speechless cartoon. All narratives were transcribed with an annotation tool designed to annotate for gesture and speech. The first results show that French children are greater talkers whereas Italian children are greater gesturers and use a lot of referential gestures, whereas American children deliver shorter narratives with fewer gestures. We observe age differences among all children on language behavior and on the gesture coding of discourse cohesion as well as on the gesture representation of events. Monologic discourse abilities and related cognitive abilities seem to occur in all populations in the same developmental stages.

\section{Les gestes et les mots : un destin lié au cours du développement langagier}

Comme cela est rappelé dans l'introduction à ce numéro de la revue Lidil, la nature multimodale (voco-verbale et gestuelle) de la communication parlée est un objet scientifique aujourd'hui reconnu aussi bien par les linguistes que par les gestualistes (Bouvet, 2001; Calbris, 2003; Kendon, 2004). La prise en compte des aspects gestuels de la communication humaine a également des répercussions sur l'étude de l'acquisition du langage.

L'époque où l'on pensait que le petit enfant, d'abord immergé dans des modes de communication non verbaux avec son entourage, devait s'affranchir de ces modes pour entrer dans la communication linguistique, est aujourd'hui révolue. Non seulement la gestualité (au sens large : gestes, mimiques, regards et postures) ne disparait pas à la fin de la période dite «pré-verbale», mais elle constitue le socle indispensable à l'apparition des moyens de communication linguistiques, comme le rappellent Aurore Batista et Marie-thérèse Le Normand dans leur article supra, et elle évolue ensuite au gré des acquisitions linguistiques et cognitives.

Ainsi, l'observation multimodale de récits spontanés ou élicités par des enfants et des adultes français (Colletta, 2009; Colletta, Pellenq et Guidetti, 2010), italiens (Graziano, 2009) et zulu (Kunene, 2010) a 
récemment apporté la preuve du fait que l'évolution des conduites langagières s'accompagne de l'évolution des conduites gestuelles. On en trouve ici une illustration dans les contributions de Djaber Fantazi et de Maria Graziano. Ces premières données factuelles nécessitent d'être complétées pour d'autres âges et d'autres tâches langagières, mais elles appellent d'ores et déjà de nouvelles questions qui sont précisément à l'origine de la recherche inter-langues dont nous présentons ci-après les premiers résultats.

\section{Culture, langue et développement discursif}

On sait depuis longtemps que la culture contraint les formes langagières dans tous leurs aspects, depuis les modes d'adresse, la forme des rituels et la réalisation des actes de langage en contexte (Saville-Troïke, 1982; Kerbrat-Orecchioni, 1994; de Nuchèze, 1998) jusqu'aux genres monologués dont font partie les textes narratifs et expositifs (Gumperz, 1982). Par ailleurs, la culture s'exprime dans les codes non verbaux de la communication, notamment dans les emblèmes (gestes conventionnels) et la mimo-gestualité accompagnant la parole (McNeill, 1992; Schneller, 1992; Morris, 1994; Kendon, 2004). Pour prendre un exemple, bien des observateurs ont noté l'habitude des Italiens à gesticuler au cours de la communication (De Jorio, 2000). Inversement, les cultures anglo-saxonnes, y compris la culture nord-américaine, sont généralement présentées comme des cultures pauvres en contact corporel et en gestualité (Barnlund, 1975). On peut donc penser que, sous l'influence de la socialisation, l'enfant de différentes cultures va mobiliser ces ressources de manière différente selon sa culture d'origine. Une étude de Iverson et al. (2008) portant sur la communication précoce montre par exemple qu'à un très jeune âge, l'enfant italien utilise davantage les gestes et dispose d'un répertoire gestuel plus important que l'enfant américain. On peut donc s'attendre à ce que son pair plus âgé utilise lui aussi davantage la gestualité que dans d'autres langues-cultures, mais cela vaut-il également pour les conduites monologuées telle la narration?

Autre facteur clé, la langue influe sur les réalisations langagières, y compris les réalisations textuelles, d'une part parce que le codage du réel diffère d'une langue à l'autre, d'autre part parce que les contraintes morphologiques et syntaxiques sont elles-mêmes variables d'une langue à l'autre. Ainsi, dans le domaine de la syntaxe, on sait qu'il existe des langues à sujet exprimé, comme l'anglais ou le français, et des langues 
à sujet nul ou non exprimé, à l'instar de l'italien ou de l'espagnol. Cette caractéristique induit des codages distincts en matière de continuité référentielle dans les usages textuels du langage, avec moins de reprises anaphoriques dans le second cas (Hickmann et Hendriks, 1999; Hickmann, 2003). Il est possible que ceci ait une incidence sur la production gestuelle, par exemple que la gestualité coverbale vienne compenser l'absence d'anaphores linguistiques lors du récit dans une langue à sujet non exprimé (Yoshioka, 2005, 2009). Si tel est le cas, autrement dit si l'hypothèse du rôle compensatoire du geste est vraie ${ }^{1}$, la gestualité coverbale produite au cours du récit dans une langue comme l'italien devrait être davantage référentielle (représentation des référents évoqués au fil du récit) que la gestualité coverbale produite par des narrateurs de langues à sujet exprimé comme le français ou l'anglais. Dans le domaine de la sémantique, on sait depuis les analyses de Talmy (1985) que les langues codent différemment l'espace et les déplacements dans l'espace. Pour coder ces derniers, les langues dites «verb-framed» comportent des verbes indiquant soit le déplacement ou la trajectoire (ex. en français : partir, revenir, monter, descendre) et d'autres indiquant le mode de déplacement (ex. en français : marcher, courir, nager, s'envoler); par contraste, dans les langues dites «satellite-framed», ces informations sont codées aussi bien par le verbe que par d'autres morphèmes, telles les prépositions en anglais (to walk to/towards / into / out $/$ down $/ . .$. ). Conséquence directe : là où une information peut être codée en une seule proposition dans une langue «satellite-framed», elle peut en nécessiter deux dans une langue «verb framed» (comparer l'anglais «he walks down the street» et le français «il descend la rue en marchant»). Or, au cours de la dernière décennie, certains travaux (Kita et Özyürek, 2003, 2007) ont mis en évidence un effet de ces codages linguistiques sur la production gestuelle.

Enregistrer un effet de la culture et/ou de la langue sur la production narrative dans ses aspects linguistiques comme dans ses aspects gestuels est certes intéressant, mais la mise en relation de ces deux facteurs avec l'âge l'est encore plus. Rappelons que l'âge constitue une variable clé pour expliquer aussi bien les habiletés langagières et textuelles enfantines que les habiletés socio-cognitives qui les sous-tendent. Le cas du récit est particulièrement intéressant de ce point de vue, car, en tant que tâche langagière monologuée, il requiert les deux types d'habiletés

1. Cette hypothèse a été formulée à diverses reprises, et dernièrement dans So, Kita et Goldin-Meadow (2009). 
(Karmiloff-Smith, 1979; Fayol, 2000). D'abord, le récit se présente comme une forme langagière contrainte à un niveau supplémentaire à celui de l'énoncé (à l'oral) ou de la phrase (à l'écrit) : le niveau du texte (Mandler et Johnson, 1977; Adam, 1992), et l'usage quotidien du langage requiert cette habileté à comprendre et produire des informations organisées à ce niveau, qu'il s'agisse de raconter un évènement ou une histoire, mais aussi de fournir une description ou une explication, ou de développer une argumentation. Ensuite, pour cette même raison, un récit présente des propriétés de cohésion et de cohérence spécifiques (Halliday et Hasan, 1976) sans équivalent dans le cours du dialogue co-construit dans l'enchainement de brefs tours de paroles, mais qui en revanche caractérisent les usages écrits du langage, de sorte que la mise en place de ces propriétés au cours de l'acquisition est une problématique commune aux aspects tardifs de l'acquisition de la parole et à l'acquisition de la lecture et de l'écriture. Enfin, l'action de raconter nécessite des capacités cognitives d'évocation (se représenter des référents absents), de contextualisation des informations linguistiques et de décentration pour adopter la perspective d'autrui, interlocuteur ou lecteur (Karmiloff-Smith, 1979; Hickmann, 2003; Tolchinsky, 2004) qui lient étroitement l'évolution des conduites narratives au développement cognitif, et permettent de comprendre pourquoi cette évolution s'étale sur des années, depuis les premiers scripts prototypiques verbalisés par l'enfant au cours du jeu symbolique jusqu'aux récits écrits de l'enfant scolarisé en cycle trois ou au collège.

Étudier la variation des conduites narratives multimodales dans un double cadre inter-langues et développemental constitue donc un réel enjeu dans la mesure où cela permet d'apprécier l'effet relatif de la culture, des contraintes linguistiques et des habiletés cognitives sur la production langagière, domaine d'investigation encore peu balisé à l'heure actuelle.

\section{Méthodologie}

Financée par l'Agence nationale de la recherche en réponse à un appel à projet fin 2005, cette recherche inter-langues implique plusieurs équipes de trois pays. Celles-ci ont d'abord conçu et élaboré un protocole de recueil de données visant à recueillir des récits parlés auprès d'enfants des deux sexes et de deux classes d'âge : de jeunes enfants âgés de 5 à 6 ans et des enfants âgés de 9 à 10 ans en fin de scolarité primaire. Avec 
ce protocole qui convenait aux deux groupes d'âge, des enfants français, italiens et américains, filles et garçons, ont ensuite été filmés en contexte péri-scolaire, à l'école mais hors de la classe, dans des tâches narratives et explicatives. Il leur était demandé de faire le récit d'un extrait d'un épisode sans parole de la série Tom et Jerry qu'ils venaient de visionner (récit contraint) et de répondre à des demandes d'explication concernant cette histoire (explications en contexte dialogué). Dans le cas présent, nous nous sommes focalisés sur la tâche narrative monologuée. Le corpus disponible est composé des récits de 153 sujets parmi lesquels 84 enfants français (corpus collectés à Grenoble et à Toulouse), 40 enfants américains et 29 enfants italiens, répartis comme suit dans les deux groupes d'âge :

\begin{tabular}{|l|c|c|c|}
\hline & 6 ans & $\mathbf{1 0}$ ans & Total \\
\hline Enfants français & 41 & 43 & 84 \\
\hline Enfants américains & 19 & 21 & 40 \\
\hline Enfants italiens & 14 & 15 & 29 \\
\hline Total & 74 & 79 & 153 \\
\hline
\end{tabular}

Tableau 1. - Répartition des sujets narrateurs par langue et par âge.

Pour exploiter ce corpus inter-langues, les équipes du projet ont eu à définir une procédure commune de transcription et d'annotation des données verbales et gestuelles. Elles ont pour cela mis au point un système d'annotation se présentant sous la forme d'une grille de codage multipistes réalisée à l'aide du logiciel $E L A N^{2}$. Un manuel de codage complète ce système d'annotation. Il présente les conventions de transcription des énoncés, adaptées du système VALIBEL ${ }^{3}$, définit les variables linguistiques et gestuelles retenues pour l'analyse, caractérise leur mode de codage, piste par piste, et fournit des exemples pour chaque variable. Le tableau 2 présente la totalité des variables analysées ainsi que les informations disponibles $(\mathrm{n}=$ nombre d'occurrences; $\%=$ proportion $;$ ratio $=$ nombre d'occurrences rapporté au nombre de propositions) pour chaque variable considérée.

2. Le logiciel ELAN est disponible en ligne à l'adresse suivante : <http://www. mpi.nl/tools/>. Une première version de ce système d'annotation a été présentée en détails dans Colletta et al. (2009).

3. Les conventions VALIBEL sont disponibles sur le site $:<\mathrm{http} / / /$ valibel.fltr. ucl.ac.be/>. 


\begin{tabular}{|c|c|}
\hline (L) Longueur du récit & $\begin{array}{l}\text { propositions }(\mathrm{n}) \\
\text { mots }(\mathrm{n})\end{array}$ \\
\hline (CS) Complexité syntaxique & $\begin{array}{l}\text { types de propositions }(n+\%) \\
\text { indices de subordination }(n+\%+\text { ratio })\end{array}$ \\
\hline (R) Rappel des évènements & $\begin{array}{l}\text { épisodes }(\mathrm{n}+\%) \\
\text { micro-épisodes }(\mathrm{n}+\%)\end{array}$ \\
\hline (CT) Cohésion textuelle & $\begin{array}{l}\text { connecteurs }(\mathrm{n}+\%+\text { ratio }) \\
\text { anaphores }(\mathrm{n}+\%+\text { ratio })\end{array}$ \\
\hline (CP) Complexité pragmatique & types d'actes $(n+\%)$ \\
\hline (PG) Production gestuelle & $\begin{array}{l}\text { gestes }(n+\text { ratio }) \\
\text { types de gestes }(n+\%)\end{array}$ \\
\hline (GR) Gestualité référentielle & $\begin{array}{l}\text { pointages abstraits }(\mathrm{n}+\text { ratio }) \\
\text { voix }(\mathrm{n}+\%+\text { ratio }) \\
\text { perspective }(\mathrm{n}+\%+\text { ratio }) \\
\text { empan gestuel }(\mathrm{n}+\%+\text { ratio })\end{array}$ \\
\hline (RGP) Relation geste-paroles & $\begin{array}{l}\text { types de combinaisons } \\
(\mathrm{n}+\%+\text { ratio })\end{array}$ \\
\hline (CE) Codage de l'espace & $\begin{array}{l}\text { linguistique }(\mathrm{n}+\%+\text { ratio }) \\
\text { gestuel }(\mathrm{n}+\%+\text { ratio })\end{array}$ \\
\hline
\end{tabular}

Tableau 2. - Liste des principales variables analysées.

Dans le cas présent, les analyses ne portent pas sur l'ensemble de ces variables, mais seulement sur les plus pertinentes du double point de vue développemental et de la comparaison inter-langues.

Au plan de la comparaison inter-langues, les différences linguistiques étant établies et connues au départ, nos hypothèses concernent essentiellement la production gestuelle. En premier lieu, comme nous l'avons signalé dans la section précédente, nous nous attendons à observer davantage de gestualité dans les récits des enfants italiens que dans les récits des enfants français, et dans les récits de ces derniers (de culture latine) que dans les récits des enfants américains (de culture anglo-saxonne). Par ailleurs, le fait que la langue italienne soit une langue à ellipse du sujet a pour conséquence que le maintien de la référence dans le récit y est moins explicitement marqué qu'en français ou en anglais. Si l'hypothèse d'une relation de compensation entre parole et gestualité est pertinente, une partie de ce marquage devrait être pris en relais par la gestualité référentielle, ce qui signifie qu'on devrait 
trouver davantage de gestualité représentationnelle dans les récits des petits Italiens. Par ailleurs, ainsi que cela a été évoqué précédemment, le codage de l'espace, et plus précisément des déplacements dans l'espace, varie d'une langue à l'autre, et ces variations ont une incidence sur la gestualité. Il est particulièrement intéressant d'étudier à partir de quel âge l'enfant perçoit les contraintes de sa langue en la matière, comme certains ont commencé à le faire (Gullberg, Hendriks et Hickmann, 2008; Özyürek et al., 2008).

Au plan développemental, une étude préalable des récits du corpus français avait permis de mettre en évidence les faits suivants : des récits plus longs, plus complexes tant au plan syntaxique qu'au plan pragmatique, plus riches en gestualité, et comportant davantage de gestes de cohésion chez les enfants de 10 ans que chez les enfants de 6 ans (les résultats sont présentés en détail dans Colletta, Pellenq et Guidetti, 2010). En toute logique, nous devrions retrouver une évolution similaire dans les corpus américains et italiens, ce qui confirmerait l'existence d'un patron de développement général des capacités discursives, déjà mis en évidence sur les seuls aspects linguistiques des textes parlés (Berman et Slobin, 1994; Hickmann, 2003), mais non encore attesté sur leur dimension gestuelle.

Par ailleurs, nous souhaitons vérifier si la production gestuelle à visée référentielle évolue effectivement, et de façon comparable dans les trois cas, en direction de l'abstraction, comme cela a été postulé par McNeill (1992) et déjà partiellement attesté ailleurs (Colletta et Pellenq, 2009; Graziano, 2009). Les variables pertinentes sont ici :

- les pointages abstraits (gestes de pointages visant à présentifier et localiser dans l'espace frontal un référent absent et à en représenter les déplacements), dont l'usage devrait croitre avec l'âge;

- la voix ou focalisation (le fait que le geste, lorsqu'il représente un personnage ou un référent animé, exprime le point de vue de l'observateur : geste $O$-vpt, ou le point de vue du personnage : geste $C$-vpt), avec l'hypothèse d'une évolution en faveur des emplois $O$-vpt car ils nécessitent de plus grandes capacités de décentration, au sens piagétien du terme (Piaget, 1967);

- la perspective (le fait que le geste $O$-vpt exécuté par le sujet révèle qu'il s'inclut - perspective interne - ou non - perspective externe - dans la scène représentée), avec une évolution en faveur de la perspective externe qui nécessite, là encore, des capacités de décentration; 
- l'empan du geste (le fait qu'un geste $C$-vpt de mime d'un personnage soit exécuté avec tout le corps ou seulement avec la main), avec l'hypothèse d'une évolution en faveur des emplois manuels, qui nécessitent d'abstraire les propriétés les plus significatives de l'action à mimer.

\section{Premiers résultats}

Précisons d'emblée que nous ne livrons ici que les tout premiers résultats de cette étude. À ce stade, nous ne disposons pas encore de toutes les données, notamment des données relatives au codage linguistique et gestuel de l'espace, qui seront traitées ailleurs et ultérieurement.

\section{Résultats de la comparaison inter-langues}

Le tableau 3 ci-après présente les résultats obtenus à partir de la comparaison inter-langues, les deux groupes d'âge confondus.

En ce qui concerne la longueur des récits (L), nous retiendrons un seul indicateur: le nombre de propositions. Le tableau 3 montre que ce sont les enfants français qui réalisent les récits les plus longs (35 propositions en moyenne) et les enfants américains qui réalisent les récits les plus brefs (13 propositions); les productions des enfants italiens se situent entre les deux (25 propositions). Les différences sont significatives $(\mathrm{F}(1,151)=26,768 ; \mathrm{p}=, 000$; notamment tests post hoc $)$ entre les récits des petits Américains et ceux des autres enfants, et l'on peut se demander pour quelles raisons ceux-ci produisent des récits nettement plus courts à partir d'une même consigne. Cela ne peut venir ni de l'âge, puisque les âges moyens sont tout-à-fait comparables (5,8 ans, 5,6 ans et 5,6 ans respectivement pour les groupes des jeunes enfants français, américains et italiens, 10 ans, 9,6 ans et 9,7 ans pour les groupes des plus âgés), ni de la consigne, qui était la même dans les trois cas. Reste donc la possibilité d'un effet du contexte de la tâche : bien qu'également effectués en contexte scolaire par des expérimentateurs non connus des enfants, les enregistrements des petits Américains ont été réalisés dans des conditions moins propices, dans des couloirs plutôt que dans des salles mises à disposition, et cela a pu déboucher sur davantage de précipitation et un moindre engagement dans la tâche. On pourrait invoquer d'autres raisons comme la sensibilité du genre aux normes culturelles et aux normes scolaires, mais il semble qu'on ne retrouve pas cet effet sur la longueur de la production dans les autres travaux inter-langues sur 
le récit (Berman et Slobin, 1994; Hickmann, 2003). Quoi qu'il en soit, il a une incidence sur d'autres variables, en particulier sur les variables gestuelles, et il nécessite de comparer les variables en tenant compte de la longueur des récits. C'est pourquoi toutes les comparaisons qui le nécessitent incluent un ratio sur le nombre de propositions en plus du nombre d'occurrences de la variable considérée.

\begin{tabular}{|c|c|c|c|c|}
\hline & Variables & FR & $\mathbf{A M}$ & IT \\
\hline$(\mathbf{L})$ & propositions (n) & 35,39 & 12,95 & 25,28 \\
\hline \multirow{2}{*}{$(\mathrm{CS})$} & propositions indépendantes $(\%)$ & 74 & 73 & 70 \\
\hline & autres propositions (\%) & 26 & 27 & 30 \\
\hline \multirow{2}{*}{ (CT) } & connecteurs (ratio sur prop.) & 1,01 & 0,92 & 0,79 \\
\hline & anaphores (ratio sur prop.) & 1,11 & 0,82 & 1,09 \\
\hline \multirow{2}{*}{$(\mathbf{C P})$} & récit (\%) & 86 & 80 & 87 \\
\hline & commentaire $(\%)$ & 14 & 20 & 13 \\
\hline \multirow{5}{*}{ (PG) } & gestes (n) & 14,73 & 6,98 & 20,07 \\
\hline & gestes (ratio) & 0,38 & 0,59 & 0,79 \\
\hline & gestualité représentationnelle (\%) & 44 & 19 & 54 \\
\hline & gestualité de cohésion et cadrage (\%) & 27 & 28 & 20 \\
\hline & gestualité non associée au récit (\%) & 29 & 53 & 26 \\
\hline \multirow{3}{*}{ (GR) } & pointages abstraits (\%) & 18 & - & 07 \\
\hline & voix : gestes $O$-vpt $(\%)$ & 58 & - & 73 \\
\hline & empan gestuel : main (\%) & 52 & - & 73 \\
\hline
\end{tabular}

Tableau 3. - Premiers résultats de la comparaison inter-langues.

Laissons de côté la complexité syntaxique (CS), la cohésion textuelle $(\mathrm{CT})$ et la complexité pragmatique $(\mathrm{CP})$, pour lesquelles nous trouvons des valeurs proches et des différences non significatives dans les trois groupes ${ }^{4}$, pour nous intéresser aux variables gestuelles (PG). Si les

4. Le nombre moins important de connecteurs dans les récits des enfants américains $(0,92$ connecteur par proposition) et surtout italiens $(0,72)$ lorsqu'on les compare aux récits en français $(1,01)$ a une cause de nature méthodologique : dans le corpus français, les enchainements de connecteurs tels «et ensuite», «donc après», "parce que avant» ont été traitées comme deux connecteurs selon la procédure décrite dans Colletta (2004, p. 236-237), alors qu'ils ont été traités comme un seul connecteur dans les récits en italien et en anglais. Quant aux anaphores, nous nous attendions à en trouver un emploi plus faible dans les récits en italien, or ce n'est pas le cas car l'ana- 
petits Français ont été les plus bavards, en revanche, comme nous nous y attendions, ce sont les petits Italiens qui ont le plus utilisé les ressources gestuelles au cours de leur récit (en moyenne 20 gestes coverbaux par récit, contre 15 pour les enfants français et 7 pour les enfants américains). Les différences sont significatives $[\mathrm{F}(1,151)=5,095 ; \mathrm{p}=, 007]$, mais seulement lorsqu'on compare les enfants italiens et les enfants américains. Pour confirmer ce résultat, il faut toutefois, comme nous l'avons expliqué plus avant, tenir compte de la longueur des récits, variable d'un groupe à l'autre. Or l'examen du ratio gestuel (nombre de gestes coverbaux par proposition) aboutit à un constat analogue $(0,79$ geste par proposition pour les enfants italiens, contre 0,59 pour les enfants américains et 0,38 pour les enfants français) et significatif $[\mathrm{F}(1,151)=12,095 ; \mathrm{p}=, 000]$ à ceci près que cette fois, les petits Américains devancent les petits Français en matière de production gestuelle. Il reste que ce résultat confirme, chez l'enfant et dans une tâche langagière monologuée, l'habitude communicative des locuteurs italiens à faire davantage de gestes en parlant.

L'examen des types de gestes employés fait apparaitre une autre différence importante, avec une gestualité plutôt représentationnelle chez les enfants italiens ( $54 \%$ des coverbaux) et français ( $44 \%$ des coverbaux), et au contraire, une gestualité peu représentationnelle chez l'enfant américain $(19 \%)$. La différence est significative $[\mathrm{F}(1,151)=14,426$; $\mathrm{p}=, 000]$, mais seulement lorsqu'on compare la proportion de gestes représentationnels des petits Américains à celle des deux autres groupes. En fait, la brièveté des récits des enfants américains induit une surreprésentation de la gestualité non directement associée au récit (53\%), et plus précisément de la gestualité performative et interactive qui accompagne les échanges qui précèdent et suivent le récit proprement dit, moments où l'adulte sollicite l'enfant, le relance ou cherche à s'assurer qu'il a terminé. En tous les cas, la gestualité des enfants italiens n'est pas significativement plus représentationnelle que celle des enfants français, et à priori, ne vient donc pas compenser le recours à l'anaphore zéro.

Enfin, les dernières comparaisons ne portent que sur la gestualité référentielle (GR) et sur les données françaises et italiennes, les données américaines n'étant pas disponibles à ce stade. Elles sont établies

phore zéro ayant aussi été codée, on y trouve au total un nombre d'anaphores comparable à celui relevé dans les autres récits, alors même que celle-ci représente $43 \%$ des emplois anaphoriques chez les enfants italiens. 
à partir de l'examen des récits des 92 enfants ayant produit des gestes à visée référentielle ( 65 enfants français et 27 enfants italiens). On trouve d'abord une différence non attendue ayant trait à l'emploi des gestes de pointage abstrait : les enfants français en produisent davantage (18\% de la gestualité référentielle) que les enfants italiens (7\%). Il semble que cette différence significative $[\mathrm{F}(1,91)=5,750 ; \mathrm{p}=, 019]$ provienne de choix de codages sensiblement différents dans les deux cas. Par contre, on ne trouve de différence significative ni en ce qui concerne la voix ou focalisation, ni en ce qui concerne l'empan gestuel.

\section{Résultats de la comparaison inter-âges}

Le tableau 4 présente les résultats les plus significatifs obtenus à partir de la comparaison des deux groupes d'âge (jeunes enfants // enfants âgés) dans les trois populations.

\begin{tabular}{|c|c|c|c|c|}
\hline & Variables & FR & $\mathbf{A M}$ & IT \\
\hline (L) & propositions (n) & $26,02 / / 44,33$ & $12,32 / / 13,52$ & $22,14 / / 28,20$ \\
\hline \multirow{2}{*}{ (CS) } & prop.indép. (\%) & $78 / / 67$ & $73 / / 73$ & $68 / / 71$ \\
\hline & autres prop. $(\%)$ & $22 / / 33$ & $27 / / 27$ & $32 / / 29$ \\
\hline \multirow{2}{*}{ (CT) } & conn. (ratio) & $1,08 / / 0,94$ & $0,72 / / 1,10$ & $0,71 / / 0,86$ \\
\hline & anaphores (ratio) & $1,03 / / 1,19$ & $0,72 / / 0,90$ & $1,01 / / 1,17$ \\
\hline \multirow{2}{*}{$(\mathbf{C P})$} & récit (\%) & $86 / / 85$ & $72 / / 88$ & $87 / / 87$ \\
\hline & commentaire $(\%)$ & $14 / / 15$ & $28 / / 12$ & $13 / / 13$ \\
\hline \multirow{6}{*}{ (PG) } & gestes (n) & $7,07 / / 22,02$ & $6,53 / / 7,38$ & $14,07 / / 25,67$ \\
\hline & gestes (ratio) & $0,28 / / 0,47$ & $0,65 / / 0,53$ & $0,66 / / 0,91$ \\
\hline & gest. référentiel. (\%) & $37 / / 51$ & $13 / / 25$ & $53 / / 55$ \\
\hline & gest. de cohésion et & & & \\
\hline & cadrage $(\%)$ & $22 / / 32$ & $23 / / 33$ & $14 / / 24$ \\
\hline & $\begin{array}{l}\text { gest. non associée au } \\
\text { récit }(\%)\end{array}$ & $41 / / 17$ & $64 / / 42$ & $33 / / 21$ \\
\hline \multirow{4}{*}{ (GR) } & pointages abstr. (\%) & $27 / / 13$ & -- & $2,5 / / 10,5$ \\
\hline & voix : gestes O-vpt & $40 / 171$ & $-z$ & $60 / / 84$ \\
\hline & $\begin{array}{l}\text { empan gestuel : main } \\
\text { emplo }\end{array}$ & $40 / / 11$ & & \\
\hline & $(\%)$ & $56 / / 49$ & -- & $64 / / 81$ \\
\hline
\end{tabular}

Tableau 4. - Premiers résultats incorporant la variable âge.

Premier constat : l'âge n'a pas partout l'effet escompté sur la longueur des récits (L). On trouve cet effet chez les enfants français, avec 
des récits de 26 propositions en moyenne à 6 ans, contre 44 à 10 ans $[\mathrm{F}(1,83)=20,520 ; \mathrm{p}=, 000]$, ainsi que, dans une moindre mesure, chez les enfants italiens, avec des récits de 22 propositions à 6 ans contre 28 à 10 ans ( $\mathrm{F}$ significatif au seuil de ,05). Par contre, on ne retrouve pas cet écart chez les enfants américains puisque les récits des plus âgés et des plus jeunes sont de même longueur. La nature très brève des récits des enfants américains de 10 ans (13 propositions en moyenne) pourrait s'expliquer par l'émergence, vers cet âge, de la capacité à résumer des faits, donc par une réinterprétation de la tâche, mais d'après des données plus complètes, celle-ci semble se produire plus tard chez l'enfant français et est loin de se traduire de façon aussi importante (Colletta, Pellenq et Guidetti, 2010). En conséquence, nous pensons que c'est le contexte de la tâche (conditions évoquées précédemment) qui a conduit le groupe des enfants américains plus âgés à abréger leur récit.

L'âge n'a pas d'effet sur la complexité syntaxique (CS), malgré un léger écart dans le corpus français, avec un taux de propositions indépendantes qui diminue (de $78 \%$ chez les plus jeunes à $67 \%$ chez les plus âgés); par ailleurs, il n'a pas d'effet non plus sur la complexité pragmatique (CP), sauf dans le corpus américain, avec un taux de propositions narratives en augmentation ${ }^{5}$. Concernant l'emploi des connecteurs et des anaphores (CT), on trouve des valeurs proches entre les deux groupes d'âge, et ce dans les trois populations, à une exception près : le taux de connecteurs par proposition augmente davantage dans les récits des jeunes Américains (il passe de 0,72 chez les 6 ans à 1,10 chez les 10 ans) ${ }^{6}$.

L'effet de l'âge est nettement plus important lorsqu'on examine les variables gestuelles (PG), en particulier lorsqu'on compare les récits des jeunes enfants français et italiens aux récits de leurs pairs plus âgés.

5. Précisons que dans le corpus français, plus complet puisque nous disposons de données fournies par des adultes, l'évolution en direction d'une syntaxe plus complexe et d'un récit plus hétérogène au plan pragmatique (avec davantage de commentaires) est surtout visible lorsqu'on compare les récits de 10 ans à ceux des adultes (Colletta, Pellenq et Guidetti, 2010). L'augmentation des propositions narratives dans le corpus américain est peut-être un épiphénomène dû à la brièveté des productions narratives collectées dans ce groupe.

6. Les causes de cet effet semblent être de nature méthodologique avec, dans l'annotation des récits américains, une définition des connecteurs élargie aux indices de régulation. 
S'agissant de la production gestuelle brute, on voit celle-ci nettement augmenter avec l'âge chez l'enfant français (de 7 à 22 gestes par récit en moyenne) et italien (de 14 à 26 gestes par récit); ces écarts sont significatifs : $\mathrm{F}(1,83)=11,142 ; \mathrm{p}=, 001$ dans le premier cas; $\mathrm{F}(1,28)=9,448$; $\mathrm{p}=, 005$ dans le second. Si l'on s'intéresse au ratio gestuel, indice qui ne tient pas compte de la différence de longueur des récits, on voit celui-ci augmenter également (de 0,28 à 0,47 gestes par proposition dans le corpus français, de 0,66 à 0,91 gestes par proposition dans le corpus italien), avec un écart significatif dans le premier cas $[\mathrm{F}(1,83)=8,576$; $\mathrm{p}=, 004]$, moins significatif dans le second $[\mathrm{F}(1,28)=5,303 ; \mathrm{p}=, 029]$. On ne retrouve pas du tout cette évolution quantitative de l'usage des ressources gestuelles dans le corpus américain, mais ce résultat est conforme à l'absence d'écart dans la longueur des récits.

L'analyse des types de gestes employés fait d'abord apparaitre une nette diminution, avec l'âge, de l'emploi des gestes non directement associés au récit, et ce dans les trois groupes linguistiques (de 41 à $17 \%$ pour les enfants français, de 64 à $42 \%$ pour les Américains, et de 33 à $21 \%$ pour les Italiens). Bien que de façon indirecte, cette évolution traduit la capacité des enfants plus âgés à mener à bien une tâche narrative monologuée sans avoir besoin d'aide (gestualité interactive) ou de relances (gestualité performative répondant aux sollicitations de l'adulte). En second lieu, la gestualité représentationnelle semble davantage utilisée à 10 ans qu'à 6 ans, là encore dans les trois groupes, mais les différences ne sont pas significatives. En revanche, lorsqu'on examine la gestualité de cohésion et de cadrage, et plus précisément les gestes de cohésion discursive (valeurs incluses dans les nombres indiqués dans le tableau), on s'aperçoit que leur emploi augmente significativement avec l'âge : il passe de 4 à $13 \%$ chez les enfants français $[\mathrm{F}(1,83)=19,427$; $\mathrm{p}=, 000]$, de 5 à $23 \%$ chez les Américains $[\mathrm{F}(1,39)=6,440 ; \mathrm{p}=, 016]$, de 8 à $23 \%$ chez les Italiens $[\mathrm{F}(1,28)=6,385 ; \mathrm{p}=, 018]$. Ce résultat est intéressant car il confirme chez l'enfant américain un phénomène déjà mis en évidence chez l'enfant français (Colletta, Pellenq et Guidetti, 2010) et italien (Graziano, 2009) : l'usage croissant, chez l'enfant, des beats ou gestes de battement, des gestes de pointage abstrait et des gestes référentiels pour structurer la progression discursive, symboliser les relations inter-propositionnelles et assurer la continuité référentielle. Ces nouveaux usages gestuels vont de pair avec le développement discursif et l'émergence de la capacité à gérer une tâche langagière monologuée, comme le montre l'évolution de l'usage des connecteurs et des anaphores également mise en évidence dans les études précitées. 
Comme pour la comparaison inter-langues, les dernières comparaisons ne portent que sur la gestualité référentielle (GR) et sur les données françaises et italiennes. En ce qui concerne les pointages abstraits, on trouve d'abord une différence certes non significative, mais contraire à notre hypothèse pour les enfants français puisque la proportion de ces gestes sur la gestualité représentationnelle est plus élevé à 6 ans (27\%) qu'à 10 ans (13\%), différence qui, pour les enfants italiens, va dans le sens attendu avec davantage de pointages abstraits $(10,5 \%)$ chez les enfants de 10 ans que chez ceux de 6 ans $(2,5 \%)^{7}$. On trouve ensuite un effet de l'âge en ce qui concerne la voix, avec l'évolution cette fois attendue en faveur de l'emploi des gestes $O$-vpt à point de vue externe. Ceux-ci passent de 40 à $71 \%$ chez les enfants français, avec un écart très significatif des variances $[\mathrm{F}(1,64)=16,171 ; \mathrm{p}=, 000]$, et de 60 à $84 \%$ chez leurs pairs italiens, avec un moindre degré de significativité $[\mathrm{F}(1,26)=5,471 ; \mathrm{p}=, 028]$. Par contre, on ne retrouve pas cette évolution vers l'abstraction au niveau de l'empan gestuel des gestes $C$ - $v p t$, puisque l'écart n'est significatif ni parmi les enfants français, ni parmi les enfants italiens alors même que les plus âgés utilisent davantage la main à des fins de mime (81\%) que les plus jeunes (64\%).

\section{Discussion et perspectives}

L'heure n'est pas à un bilan définitif de cette étude, puisque toutes les données n'ont pas encore été traitées. Mais voyons ce qu'il en ressort à ce stade pour chacun de ses deux volets.

La comparaison inter-langues montre que ce sont les enfants français qui réalisent les récits les plus longs, mais que ce sont bel et bien les enfants italiens qui, conformément à nos attentes, utilisent le plus les ressources gestuelles à des fins narratives. La propension des adultes italiens à faire des gestes en parlant se vérifie donc ici chez l'enfant, dans une tâche langagière monologuée. Pour le reste, on note peu de différences entre les productions linguistiques et gestuelles des trois

7. L'identification des pointages abstraits constitue une réelle difficulté car elle est indissolublement liée à celle de l'identification des anaphores gestuelles. Les choix méthodologiques s'avèrent donc cruciaux, et dans le cas présent, les équipes française et italienne ont procédé à partir de définitions distinctes. Des analyses plus récentes (Reig Alamillo et al., sous presse; Capirci et al., 2010) ont permis de corriger et affiner ces résultats. 
groupes, y compris en ce qui concerne la gestualité représentationnelle. Rappelons qu'on s'attendait à voir cette dernière davantage représentée dans les productions narratives des enfants italiens, eu égard au fait que le maintien de la référence dans le récit est moins explicitement marqué en italien qu'en français ou en anglais. Or nos données ne vont pas dans ce sens et ne semblent donc pas supporter la thèse d'une compensation des ressources linguistiques par les ressources gestuelles, à tout le moins dans le contexte de cette tâche. Une autre entrée intéressante sur cette question des relations gestes-paroles est celle du codage de l'espace, dont on sait qu'il varie d'une langue à l'autre. Mais ce sont les données américaines qui s'avèrent ici cruciales, car l'anglais s'oppose sur ce point au français et à l'italien. Le traitement de ces données est en voie de réalisation et nous permettra prochainement de compléter les résultats actuels.

La comparaison inter-âges fournit plusieurs résultats intéressants. L'évolution attendue des productions narratives est, au plan linguistique et d'un point de vue quantitatif, surtout perceptible dans la longueur des récits (si on fait abstraction des récits du corpus américain, atypiques en raison de leurs conditions de production). Les évolutions qualitatives, qu'elles concernent la syntaxe, la cohésion discursive ou la dimension pragmatique de l'activité narrative, sont encore peu apparentes entre les âges de 6 et 10 ans (alors même qu'elles sont effectives au-delà de cet âge, comme nous l'avons indiqué à plusieurs reprises). En revanche, entre 6 et 10 ans, il se passe déjà beaucoup de choses au plan de la gestualité, comme le montre de façon nette l'accroissement de l'emploi des coverbaux dans les groupes des enfants les plus âgés (si on fait abstraction, là encore, des récits du corpus américain, atypiques de ce point de vue). Par ailleurs, on note de réelles évolutions qualitatives, les unes (la moindre part de la gestualité non narrative et le recours croissant à la gestualité de cohésion) accompagnant le développement discursif et attestant de la maitrise croissante des usages monologués de la parole, les autres (le recours croissant à la gestualité $O$-vpt) accompagnant le développement cognitif et témoignant de plus grandes capacités d'abstraction.

À ce stade, le plus intéressant à retenir est donc l'existence d'évolutions qui affectent aussi bien les aspects linguistiques que les aspects gestuels des productions narratives, et qui paraissent se produire de manière similaire d'une langue-culture à l'autre. Malgré les différences inhérentes aux langues et aux variations culturelles en matière de gestualité, il semble que l'évolution des capacités discursives et des capa- 
cités cognitives sous-jacentes soit semblable dans les trois cas, et que nous ayons affaire à un patron développemental commun pour le récit parlé multimodal.

L'exploitation continuée de ces données nous permettra de préciser l'image de ce tableau évolutif et, nous l'espérons, de déboucher sur des hypothèses plus précises quant aux relations entre acquisitions linguistiques et développement cognitif dans l'évolution des capacités discursives multimodales chez l'enfant.

\section{Remerciements}

Les données récoltées pour cette étude ont pu l'être grâce à un financement par l'ANR en 2005 (projet ANR-05-BLAN-0178). Nous remercions Asela Reig Alamillo pour son aide dans le traitement des données. Nous remercions également les enfants de Grenoble, Toulouse, Chicago et Rome qui ont accepté de collaborer à cette étude.

\section{RÉFÉRENCES BIBLIOGRAPHIQUES}

AdAm J.-M. (1992) : Les textes : types et prototypes. Récit, description, argumentation, explication, dialogue, Paris, Nathan.

B ARNLUND D. C. (1975) : "Communicative style in two cultures», dans A. Kendon, R. M. Harris, M. R. Key (éds), Organization in behaviour in face-to-face interaction, La Haye, Mouton, p. 427-456.

Berman R. A. et Slobin D. I. (1994) : Relating Events in Narrative: A Crosslinguistic Developmental Study, Hillsdale, Lawrence Erlbaum Associates.

Bouvet D. (2001) : La dimension corporelle de la parole, Paris, Peeters.

CALBRIS G. (2003) : L'expression gestuelle de la pensée d'un homme politique, Paris, CNRS Éditions.

Capirci O., Colletta J.-M., Cristilli C., De Angelis V. et Graziano M. (2010) : «Linguistic and gestural reference tracking in Italian and French children's narratives», communication présentée dans le cadre de la 4th Conference of the International Society for Gesture Studies (ISGS) (25-30 juillet 2010, European University Viadrina, Francfortsur-l'Oder).

Colletta J.-M. (2004) : Le développement de la parole chez l'enfant âgé de 6 à 11 ans, Hayen (Belgique), Mardaga.

Colletta J.-M. (2009) : «Comparative analysis of children's narratives at different ages: a multimodal approach», Gesture, vol. 9, nº 1, p. 61-97. 
Colletta J.-M., Kunene R. N., Venouil A., Kaufmann V. et Simon J.-P. (2009) : «Multitrack annotation of child language and gestures», dans M. Kipp, J.-C. Martin, P. Paggio et D. Heylen (éds), Multimodal Corpora, From Models of Natural Interactions to Systems and Applications, LNAI 5509, Berlin, Springer, p. 54-72.

Colletta J.-M. et PellenQ C. (2009) : «Multimodal explanations in French children aged from 3 to 11 years », dans N. Nippold et C. Scott (éds), Expository Discourse in Children, Adolescents, and Adults. Development and Disorders, New York, Psychology Press, Erlbaum, Taylor \& Francis, p. 63-97.

Colletta J.-M., PellenQ C. et Guidetti M. (2010) : «Age-related changes in co-speech gesture and narrative: Evidence from French children and adults », Speech Comm, vol. 52, n 6, p. 565-576.

De Jorio A. (2000) : Gesture in Naples and Gesture in Classical Antiquity, A translation of La mimica degli antichi investigata nel gestire napoletano (1832), and with an Introduction and Notes, by Adam Kendon, Bloomington, Indiana Press.

Fayol M. (2000) : «Comprendre et produire des textes écrits : 1'exemple du récit», dans M. Kail et M. Fayol, L'acquisition du langage. T. 2 : Le langage en développement. Au-delà de trois ans, Paris, Presses universitaires de France, p. 183-213.

Graziano M. (2009) : Le développement de la relation entre les compétences verbale et gestuelle dans la construction d'un texte narratif chez l'enfant âgé de 4 à 10 ans, thèse de doctorat, Université de Grenoble et Università degli Studî Suor Orsola Benincasa (Naples).

Gullberg M., Hendricks H. et Hickmann M. (2008) : «Learning to talk and gesture about motion in French», First Language, vol. 28, n ${ }^{\circ} 2$, p. 200-236.

Gumperz J. J. (1982) : Discourse strategies, Cambridge, Cambridge University Press.

Halliday M. A. K. et Hasan R. (1976) : Cohesion in English, Londres, Longman.

Hickmann M. (2003) : Children's Discourse: Person, Space and Time across Languages, Cambridge, Cambridge University Press.

Hickmann M. et Hendricks H. (1999) : «Cohesion and anaphora in children's narratives: a comparison of English, French, German, and Mandarin Chinese », Journal of Child Language, vol. 26, n 2, p. 419-452.

Iverson J. M., Capirci O., Volterra V. et Goldin-Meadow S. (2008) : «Learning to talk in a gesture-rich world: Early communication in Italian vs. American children», First Language, vol. 28, n 2, p. 164-181.

Karmiloff-Smith A. (1979) : A functional approach to child language, Cambridge, Cambridge University Press. 
Kendon A. (2004) : Gesture. Visible action as utterance, Cambridge, Cambridge University Press.

Kerbrat-Orecchioni C. (1994) : Les interactions verbales, t. 3, Paris, Armand Colin.

KitA S. et ÖZYÜREK A. (2003) : «What does cross-linguistic variation in semantic coordination of speech and gesture reveal? Evidence for an interface representation of spatial thinking and speaking », Journal of Memory and Language, vol. 48, $\mathrm{n}^{\circ} 1$, p. 16-32.

KITA S. et ÖZYÜREK A. (2007) : «How does spoken language shape iconic gestures », dans S. Duncan, J. Cassell et E. T. Levy (éds), Gesture and the Dynamic Dimension of Language: Essays in Honor of David McNeill, Amsterdam, John Benjamins, p. 67-74.

KunENE R. N. (2010) : A comparative study of the development of multimodal narratives in French and Zulu children and adults, these de doctorat, Université de Grenoble.

Mandler J. M. et Johnson N. S. (1977) : «Remembrance of things parsed: Story structure and recall», Cognitive psychology, n 9, p. 111-151.

MCNeILl D. (1992) : Hand and mind. What gestures reveal about thought, Chicago, University of Chicago Press.

Morris D. (1994) : Body talk. A world guide to gestures, Londres, Jonathan Cape.

NuchÈze (DE) V. (1998): Sous les discours, l'interaction, Paris, L'Harmattan.

Özyürek A., Kita S., Allen S., Brown A., Furman R. et Ishizuka T. (2008) : «Development of cross-linguistic variation in speech and gesture: motion events in English and Turkish», Developmental Psychology, vol. 44, no 4, p. 1040-1054.

Piaget J. (1967, éd. orig. 1937) : La construction du réel chez l'enfant, Neuchâtel, Delachaux et Niestlé.

Reig Alamillo A., Colletta J.-M. et Kunene R. N. (sous presse) : «Reference tracking in gesture and speech. A developmental study on French narratives », Rivista di Psicolinguistica Applicata (RIPLA).

Saville-Troike M. (1982) : The ethnography of communication, An introduction, Oxford, Basil Blackwell.

SCHNELleR R. (1992) : «Many gestures, many meanings: Nonverbal diversity in Israel», dans F. Poyatos (éd.), Advances of nonverbal communication, Amsterdam, John Benjamins, p. 213-233.

So W. Ch., Kita S. et Goldin-Meadow S. (2009) : «Using hands to identify who does what to whom: Gesture and speech go hand-in-hand», Cognitive Science, vol. 33, n 1, p. 115-125.

TALMY L. (1985) : «Lexicalisation patterns: semantic structures in lexical forms », dans Language Typology and Syntactic Description. Vol. 3: Grammatical Categories and the lexicon, Cambridge, Cambridge University Press, p. 57-149. 
Tolchinsky L. (2004) : «The nature and scope of later language development», dans R. A. Berman (éd.), Language Development across Childhood and Adolescence, Amsterdam, John Benjamins, p. 233-247.

Yoshioka K. (2005) : «Linguistic and gestural introduction and tracking of referents in L1 and L2 discourse», Groningen Dissertations in Linguistics, $\mathrm{n}^{\circ} 55$.

Yoshioka K. (2009) : «Gesture and information structure in first and second language », Gesture, vol. 8, n 2, p. 236-255. 